\title{
ESTUDIO DE LA DIABETES MELLITUS EN ESTUDIANTES DE LA ESCUELA PROFESIONAL DE CIENCIAS FARMACEUTICAS Y BIOQUIMMICA
}

\section{STUDY OF DIABETES MELLITUS IN STUDENTS OF THE PROFESSIONAL SCHOOL OF PHARMACEUTICAL SCIENCES AND BIOCHEMISTRY.}

\author{
AUTORES: Dra. Q.F. Diana Esmeralda Andamayo Flores, Mg. Q.F. Vilma Amparo Junchaya Yllescas, ${ }^{2}$ \\ Mg. T.M. Renee Soledad Orrego Cabanillas, ${ }^{3}$ Mg. Q.F. Martha Raquel Valderrama Sueldo, \\ Mg. Freedy Dante Orihuela Villar, ${ }^{5}$ M.C. Hector Millan Camposano, Dra. C.D. Diana Esmeralda \\ Castillo Andamayo, Est. Yorsi Janet Lambert Rios, ${ }^{8}$ Est. Katy Roxana Paucar Alarcon?
}

\section{RESUMEN}

El trabajo de investigación titulado "Estudio de la diabetes mellitus en estudiantes de la Escuela Profesional de Ciencias Farmacéuticas y Bioquímica" tuvo como objetivo principal Determinar la Prevalencia de Diabetes Mellitus en los estudiantes de Ciencias Farmacéuticas y Bioquímica del semestre académico 2016 - I. El método empleado para realizar el trabajo de investigación fue el método científico, el tipo de investigación fue básica, el nivel de investigación fue descriptivo; el diseño de la investigación fue experimental transversal. La investigación fue realizada en una muestra de 242 estudiantes de la Escuela Profesional de Ciencias Farmacéuticas y Bioquímica; es así que se tuvo como resultado que de 242 personas evaluadas, el $93.4 \%$ presentan la glucosa basal $<110 \mathrm{mg} / \mathrm{dl}$ siendo calificados como "Normal" y el $6.6 \%$ presentan su glucosa basal de 110- $126 \mathrm{mg} / \mathrm{dl}$ calificados como "Intolerante A La Glucosa". Asimismo, se concluye que los 242 estudiantes a los cuales se les realizo el análisis de glicemia capilar ninguno presenta diabetes mellitus.

\section{PALABRA CLAVE:}

Diabetes, glicemia, glucosa.

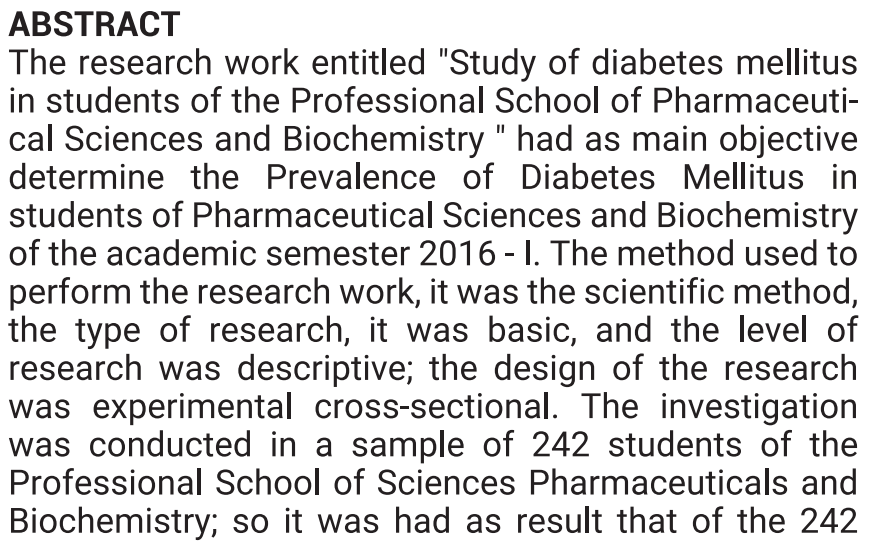

people evaluated, $93.4 \%$ present baseline glucose $<110$ $\mathrm{mg} / \mathrm{dl}$ being rated as "Normal" and $6.6 \%$ present their glucose baseline of $110-126 \mathrm{mg} / \mathrm{dl}$ qualified as "Intolerant a Glucose". Likewise, it is concluded that of the 242 students who were analyzed for capillary glycaemia none presents diabetes mellitus.

\section{KEYWORDS:}

Diabetes mellitus, glycaemia and glucose.

\section{INTRODUCCIÓN}

Sobre las márgenes del indo (India), en el siglo V A.C. sushuruta, descubre el sabor dulce de la orina que atraía a los insectos y a las hormigas, (la orina de miel) asociada con la sed, debilitamiento muscular, somnolencia, dificultad para respirar, es decir, la clínica de la descompensación y la acidosis, encontraba la enfermedad en los obesos y ricos. En un nuevo juego de épocas aparecen los griegos, Apolonio de Mileto 200 A.C. y Demetrio de Apamea (300-200) A.C. a los que se les atribuye haber introducido la denominación Diabetes. Areteo alrededor del año 131 escribe. "el desarrollo de la enfermedad es gradual, pero de corta vida en quién ésta se desarrolle"; el deseo de beber se hace cada vez más fuerte y cuando la enfermedad está en su punto más elevado orinan constantemente de ese hecho ha derivado su nombre, por lo que diabetes significa sifón. Galeno posteriormente lo atribuyó a la incapacidad del riñón para retener agua. Thomas Willis, en 1674, describe que el azúcar estaba primero en la sangre y de allí pasaba a la orina y Cullen, 1776, le dio el nombre de Mellitus diferenciándola de la diabetes insípida.

La glucosa en la sangre fue determinada por primera vez, en 1859, por Claude Bernard quien señaló que la hiperglicemia era el signo fundamental de la enfermedad. Diez años después, Langerhans describió los islotes pancreáticos, que posteriormente recibieron su nombre en el año 1889. Los científicos Minkowsky y 
Von Mering, en 1889, encontraron que el origen de la Diabetes era pancreático y no renal. En 1901 Opie le atribuyó el origen a alteraciones de los islotes de Langerhans. Al inicio del siglo XX Zuelser y Paulesco demostraron que un extracto pancreático era capaz de descender la glucemia, pero sus investigaciones no fueron difundidas.

Solo hasta 1921 en Toronto, Canadá, Frederic Banting y Charles Best el primero un ortopedista y el segundo un estudiante de medicina, trabajando en los laboratorios del Dr. JJR Macleod, encontraron un extracto, el cual inyectado en perros pancreatectomizados producía una disminución de la glucosa circulante; este extracto inicialmente lo denominaron isletina pero luego lo bautizaron como insulina.

La Diabetes Mellitus es una enfermedad crónica, considerada actualmente como un problema de salud pública. Su atención se centra tanto en la prevención de su aparición como en mejorar su control, tratamiento y pronóstico. La Diabetes, que se inicia como un envejecimiento prematuro, puede desarrollar devastadoras complicaciones en los pacientes y producir un impacto socioeconómico importante a nivel mundial, con aumento del costo tanto personal como social, no sólo en su tratamiento sino también en la pérdida de años de vida útil.

La prevalencia mundial de la Diabetes Mellitus (DM) se ha incrementado en grado impresionante durante los dos últimos decenios, por lo menos 171 millones de personas en el mundo sufren de Diabetes y se proyecta que las cifras aumentarán a 300 millones para el año 2025. Aunque la prevalencia de la DM tipo 1 y tipo 2 está aumentando en todo el mundo, cabe esperar que la del tipo 2 aumente con más rapidez en el futuro por la obesidad creciente y la reducción de la actividad física.

Por todo lo expuesto la presente investigación tendrá como objetivo general "Determinar Diabetes Mellitus en los estudiantes de Ciencias Farmacéuticas y Bioquímica del semestre académico 2016 - I".

\section{OBJETIVO DE LA INVESTIGACIÓN OBJETIVO GENERAL.}

Determinar la Prevalencia de Diabetes Mellitus en los estudiantes de Ciencias Farmacéuticas y Bioquímica del semestre académico 2016 - I.

\section{OBJETIVOS ESPECÍFICOS}

- Distribuir según sexo y edad a los estudiantes de Ciencias Farmacéuticas y Bioquímica que presentan aumento de la glicemia capilar.

- Determinar los niveles de glicemia capilar como diagnóstico de Diabetes Mellitus tipo 2.

\section{MATERIALES}

La investigación se realizó a través de hojas de recolección de datos previa autorización de cada uno de los estudiantes que acudió al área de estudio establecido.

\section{METODOLOGÍA}

MÉTODO DE LA INVESTIGACIÓN: El presente trabajo de investigación se ha diseñado a partir de los siguientes pasos que se describe en el método científico que según Tamayo, Tamayo nos dice que "Es un procedimiento para descubrir las condiciones en que se presentan sucesos específicos caracterizados generalmente por ser tentativo, verificable, de razonamiento riguroso y observación empírica".

TIPO Y NIVEL DE INVESTIGACIÓN: Tipo de investigación del presente, trabajo es considerado Básico, porque el autor menciona que: "Es la búsqueda de nuevos conocimientos y campos de investigación, recoge información de la realidad para enriquecer el conocimiento científico, orientando al descubrimiento de principios y leyes; busca el progreso científico, acrecentar los conocimientos teóricos; busca desarrollar una teoría o modelo teórico científico basado en principios y leyes".

Con relación al nivel de investigación del presente trabajo, es considerado descriptivo, porque se definen variables y esto consta de una sola variable.

Diseño De La Investigación: No experimental, transversal descriptivo.

POBLACIÓN DE ESTUDIO: Estuvo integrada por 612 estudiantes de Ciencias Farmacéuticas y Bioquímica de la universidad privada de Huancayo "Franklin Roosevelt".

MUESTRA: De Tipo no probabilística por conveniencia y se aplicará a todos los involucrados.

Los mismos debieron cumplir con los criterios de inclusión y exclusión propuestos.

Estará Conformada por 242 estudiantes de Ciencias Farmacéuticas y Bioquímica de la Universidad Privada de Huancayo "Franklin Roosevelt" escogidas de acuerdo al diseño de muestra, donde fueron escogidos al azar.

\section{RESULTADOS}

\section{Cuadro 1. Prevalencia de diabetes}

\begin{tabular}{|c|c|c|c|c|}
\hline & $\begin{array}{l}\text { FRECUEN } \\
\text { CIA }\end{array}$ & PORCENTAJE & $\begin{array}{l}\text { PORCENTAJE } \\
\text { VÁLIDO }\end{array}$ & $\begin{array}{c}\text { PORCENTAJE } \\
\text { ACUMULADO }\end{array}$ \\
\hline$<110$ "normal" & 226 & $93.4 \%$ & $93.4 \%$ & $93.4 \%$ \\
\hline $\begin{array}{c}110-126 \\
\text { "intolerante a la } \\
\text { glucosa" }\end{array}$ & 16 & $6.6 \%$ & $6.6 \%$ & 100 \\
\hline total & $\mathbf{2 4 2}$ & $\mathbf{1 0 0 \%}$ & $\mathbf{1 0 0 \%}$ & \\
\hline
\end{tabular}

Fuente: Universidad Privada de Huancayo "Franklin Roosevelt"

GRAFICO 1: Resultado de la prevalencia de diabetes 


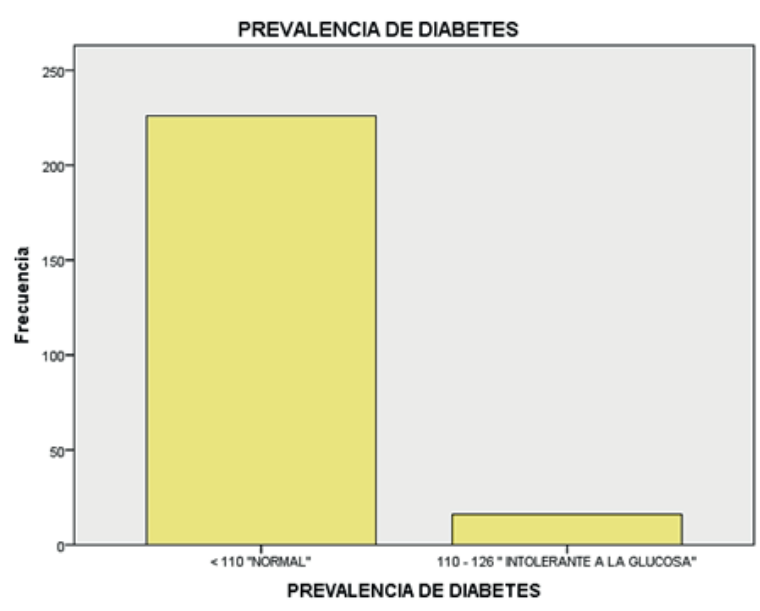

Fuente: Universidad Privada de Huancayo "Franklin Roosevelt"

INTERPRETACIÓN: de los 242 personas evaluadas, el $93.4 \%$ presentan la glucosa basal $<110 \mathrm{mg} / \mathrm{dl}$ siendo calificados como "Normal" y el $6.6 \%$ presentan su glucosa basal de $110-126 \mathrm{mg} / \mathrm{dl}$ calificados como "Intolerante A La Glucosa".

Cuadro $N^{\circ}$ 2: Prevalencia de diabetes mellitus en relación con la edad

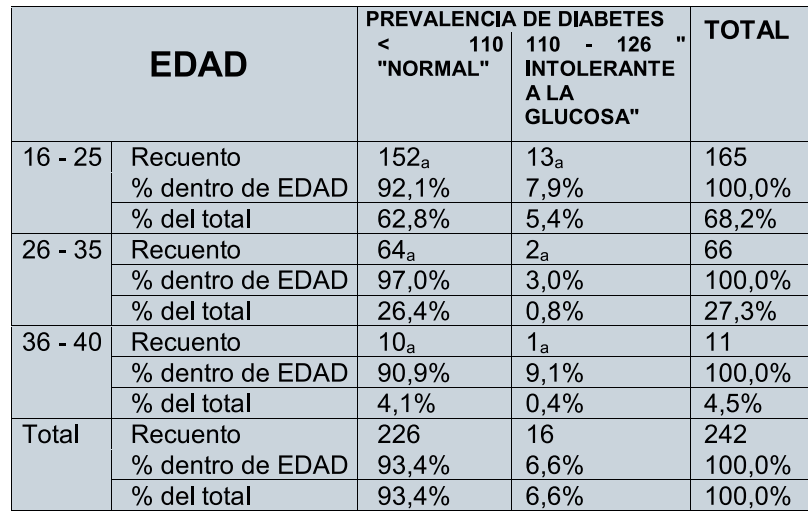

Fuente: Universidad Privada de Huancayo "Franklin Roosevelt"

\section{GRAFICO N²: Resultados edad}

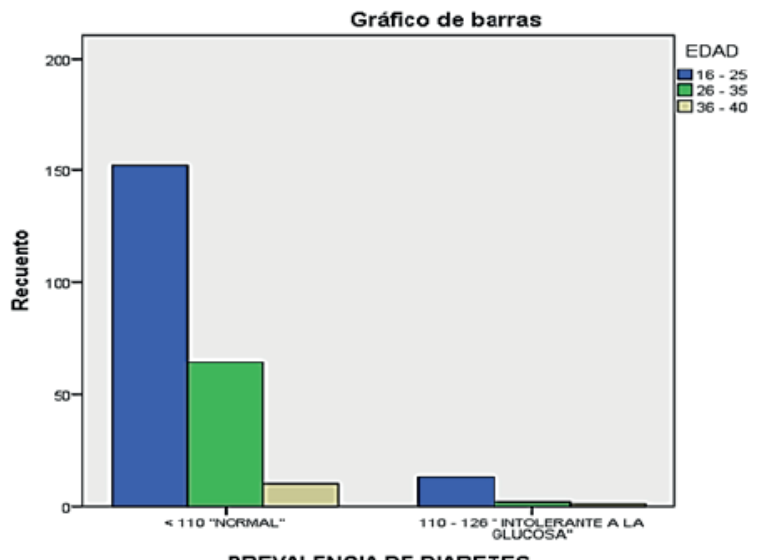

PREVALENCIA DE DIABETES
INTERPRETACIÓN: La edad predominante es la 16 - 25 años en un $62.8 \%$, su glucosa basal es $<110 \mathrm{mg} / \mathrm{dl}$ "Normal" y este mismo grupo etario tiene el mayor porcentaje de "Intolerante A La Glucosa" representa el $4.5 \%$.

Cuadro $\mathrm{N}^{\circ}$ 3: Prevalencia de diabetes mellitus en relación con el sexo

\begin{tabular}{|l|l|l|l|}
\hline \multicolumn{2}{|c|}{ SEXO } & \multicolumn{2}{|c|}{ PREVALENCIA DE DIABETES } \\
\cline { 3 - 4 } & $\begin{array}{l}<110 \\
\text { "NORMAL" }\end{array}$ & $\begin{array}{l}\mathbf{1 1 0}-126 " \\
\text { INTOLERANTE A } \\
\text { LA GLUCOSA" }\end{array}$ \\
\hline \multirow{2}{*}{ FEMENINO } & Recuento & $213 \mathrm{a}$ & $14 \mathrm{a}$ \\
\cline { 3 - 4 } & $\%$ dentro de SEXO & $93,8 \%$ & $6,2 \%$ \\
\cline { 2 - 4 } & $\%$ del total & $88,0 \%$ & $5,8 \%$ \\
\hline \multirow{3}{*}{ MASCULINO } & Recuento & $13 \mathrm{a}$ & $2 \mathrm{a}$ \\
\cline { 2 - 4 } & $\%$ dentro de SEXO & $86,7 \%$ & $13,3 \%$ \\
\cline { 2 - 4 } & $\%$ del total & $5,4 \%$ & $0,8 \%$ \\
\hline \multirow{3}{*}{ TOTAL } & Recuento & 226 & 16 \\
\cline { 2 - 4 } & $\%$ dentro de SEXO & $93,4 \%$ & $6,6 \%$ \\
\cline { 2 - 4 } & $\%$ del total & $93,4 \%$ & $6,6 \%$ \\
\hline
\end{tabular}

Fuente: Universidad Privada de Huancayo "Franklin Roosevelt"

GRAFICO N`3: Resultado de prevalencia de diabetes mellitus con el sexo

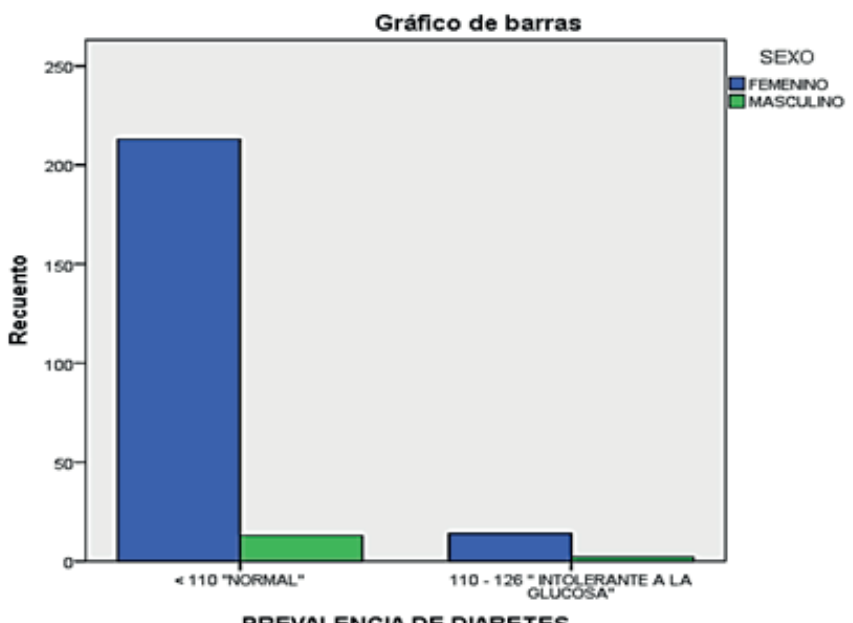

PREVALENCIA DE DIABETES

INTERPRETACIÓN: El sexo predominante es el femenino con un $93.8 \%$ su glicemia se encuentra normal y $5.8 \%$ se encuentra como intolerante a la glucosa. Seguidamente el sexo masculino con un $86.7 \%$ su glicemia es normal y un $0.8 \%$ se encuentra como intolerante a la glucosa.

\section{DISCUSIÓN}

- La Diabetes Mellitus es un problema mundial de salud pública. En algunas áreas de América la población no tiene información acerca de la Diabetes Mellitus. Se sabe que la tendencia es ir incrementándose cada vez la prevalencia de Diabetes Mellitus en un futuro inmediato. 
En el trabajo de investigación encontramos la glicemia capilar con mayor porcentaje de $93.4 \%$ con valores normales es decir se encuentran menor a $110 \mathrm{mg} / \mathrm{dl}$ y un $6.6 \%$ su glicemia se encuentra de $110-126 \mathrm{mg} / \mathrm{dl}$ son del grupo de intolerantes a la glucosa, son un grupo de personas que están predispuestas a padecer de diabetes en comparación Rosello, M. y cols. Solo obtuvo un $1.3 \%$ como ITG. Finalmente, en el trabajo realizado no se obtuvo ningún resultado que su glicemia haya sido > $126 \mathrm{mg} / \mathrm{dl}$ en comparación de Rosello, M. y cols que obtuvo como resultado $0.2 \%$ diabetes provisional. En los datos obtenidos se evidencian datos que, no se asemejan a la referencia.

La edad predominante es la 16 - 25 años en un $62.8 \%$, su glucosa basal es $<110 \mathrm{mg} / \mathrm{dl}$ "Normal" y grupo etario de 16- 25 años su glucosa basal es $110-126 \mathrm{mg} / \mathrm{dl}$ "intolerante a la glucosa" representa el $4.5 \%$ que en comparación con González, $\mathbf{J}$ y cols. Observó un incremento en la prevalencia de $8.8 \%$ a $16.6 \%$ en el grupo de 40 a 49 años, como se puede observar en los datos el resultado del trabajo el grupo etario con mayor predisposición a la diabetes es de 16 - 25 años que corroboran la investigación realizada.

También se encontró en el estudio dentro de lo que respecta al sexo predomina con alto porcentaje de riesgo a la diabetes es del sexo femenino con un $5.8 \%$ que son categorizados como Intolerantes A La Glucosa. En comparación con González, J y cols, también el género femenino predomina con mayor predisposición al sobrepeso y obesidad. En ambos géneros se obtuvieron valores del Índice Cintura Cadera (ICC) en niveles de riesgo, sobre todo en los grupos de edades más jóvenes.

\section{CONCLUSIONES}

1. De los 242 estudiantes que se les realizo el análisis de glicemia capilar ninguno presenta diabetes mellitus obteniendo como resultado 226 con un porcentaje de $93.4 \%$ estudiantes tienen la glucosa $<110 \mathrm{mg} / \mathrm{dl}$ categorizados como "NORMAL" y 16 estudiantes con un porcentaje de $6.6 \%$ estudiantes tienen la glucosa 110 - $126 \mathrm{mg} / \mathrm{dl}$ categorizados como "INTOLERANTES A LA GLUCOSA".

- De acuerdo con los resultados obtenidos se evidencia que ambos sexos presentan aumento de glicemia capilar que se encuentra entre los valores de $110-126$ $\mathrm{mg} / \mathrm{dl}$, teniendo como referencia que lo normal de glicemia en ayunas es de $70-110 \mathrm{mg} / \mathrm{dl}$. Pero siendo el sexo femenino con $5.8 \%$ y el grupo etario de $16-25$ años con $4,5 \%$ son los que tienen mayor predisposición a la diabetes "Intolerantes A La Glucosa".

\section{REFERENCIAS BIBLIOGRÁFIA}

1. American Diabetes Association. Report of the expert commitee on the diagnosis y classification of Diabetes Mellitus; 1997. 20: 1183-1197.

2. Hernandez R, Fernandez C, Baptista P. Metodología de la investigación. 6ta ed. México: Editorial Mc Grawl Hill; 2014. Pp.80.

3. García, F y cols. Prevalencia de Diabetes Mellitus y Factores de riesgo relacionados en una población urbana. Revista de medicina interna. Vol. 20. Perú; 2001.

4. Gasteiz V. Métodos Diagnósticos para Diabetes Mellitus. Guía práctica clínica sobre Diabetes Tipo 2. Madrid; 2008.

5. González J y cols. Valoración de factores de riesgo para Diabetes Mellitus tipo 2 en una comunidad semiurbana de la ciudad de México. Revista Médica. Volumen 12. $N^{\circ} 4$.

Fecha de Recepción: 15/12/2016

Fecha de Revisión: 30/12/2016

Correo Electronico: dandamayo@uroosevelt.edu.pe 